\title{
A CRITICAL ASSESSMENT OF MODELS PREDICTING ALLOYING BEHAVIOUR BY MEANS OF PATTERN RECOGNITION
}

\author{
A. P. M. KENTGENS \\ Institute for Theoretical Physics, Catholic University, Toernooiveld, 6525 ED Nijmegen \\ (The Netherlands)
}

F. W. PIJPERS*

Department of Analytical Chemistry, Catholic University, Toernooiveld, 6525 ED

Nijmegen (The Netherlands)

G. VERTOGEN

Institute for Theoretical Physics, Catholic University, Toernooiveld, 6525 ED Nijmegen (The Netherlands)

(Received 29th October 1982)

\section{SUMMARY}

Pattern recognition techniques are applied in order to investigate the relevance of a large set of physical and chemical parameters for the description of the formation of binary alloys. Only alloys with a well-established sign of the heat of formation are considered. The electronic work function and the electron density at the boundary of the Wigner-Seitz cell, as given by Miedema et al., appear to be the most relevant parameters. A difference between the Miedema scale and the Pauling electronegativity scale is noted. The parameters suggested by Miedema et al. are evaluated.

The possible prediction of the alloying behaviour of two metals has intrigued many people. Several empirical rules have been proposed in order to predict such behaviour. The Hume-Rothery rule is well known [1]; this " $15 \%$ rule" states that two metals do not form a solid solution if their atomic radii differ by over $15 \%$. Further, the stability of intermetallic compounds increases with increasing difference in electronegativity between the constituent elements, and the concept of "valence" must also be taken into account [1]. Waber et al. [2] showed that the $15 \%$ rule, in combination with a second rule stating that the difference in electronegativity must not exceed 0.4 Pauling electronegativity units [3], predicts the mutual solubility of two metals with an accuracy of $77 \%$. It should be noted that alloying refers both to the mutual solubility of two metals (i.e., random distribution of atoms over the crystal lattice in the solid phase) and to the formation of intermetallic compounds (i.e., regular distribution on the crystal lattice). A slightly different approach was given by Mott [4]; combining the Pauling [3] term for ionic bonds with Hildebrandt's solubility parameter, Mott predicted the mutual solubility of two liquid metals with an accuracy of $80 \%$. 
By far the most successful approach is that given by Miedema et al. [5]. Their empirical model, which appears to be related to Mott's model, is based on a "macroscopic atom picture". Its range of validity covers liquid as well as solid alloys of a transition metal with another transition metal, or a noble metal, or an alkali or alkaline-earth metal; the model also covers liquid alloys consisting of two non-transition metals. An exceptional situation arises for alloys of a transition metal with " $p$-type" metals [5] (see Table 2), where additional energy effects occur; such alloys could also be covered by adding an extra term to the model. This type of alloy, however, will not be considered in this paper.

The model of Miedema et al. considers an alloy to consist of atomic (Wigner-Seitz) cells of the individual elements. The basic idea is that contact between Wigner-Seitz cells of two different elements has two effects: first, charge transfer towards the most electronegative cell results in a negative contribution to the energy; second, elimination of the accompanying discontinuity in the electron density at the boundary of the cells contributes positively to the energy.

Sophisticated theories based on quantum mechanics have been presented in order to explain alloying behaviour [6-9]. These theories, however, cannot account for the impressive overall applicability of the semi-empirical model [5]. Even the physical picture proposed by Miedema et al. has been criticized by Williams et al. [10]. Undoubtedly, a fundamental theory should provide a physical understanding of the parameters introduced by Miedema et al. Before such a theory is formulated, it is clearly of interest to know which physical and chemical parameters play a prominent part in alloying, and to assess if the parameters of Miedema et al. are the only relevant ones.

The purpose of this paper is to discuss alloying behaviour from the point of view of pattern recognition [11-13]. This technique selects the relevant parameters from a set of parameters under investigation; the ARTHUR computer program [14] is commonly used. A concise description of the techniques relevant to this paper is given below. This is followed by an argument assuming that the description of the generally accepted electronegativity difference given by Miedema et al. is correct. Replacement of the less well understood term describing the difference in electron density by a more satisfactory physical or chemical parameter is then discussed and the Miedema work function scale and the Pauling electronegativity scale [3] are compared. Miedema et al. [5] describe the charge transfer in an alloy by means of a work function scale for pure metals, specially designed for the purpose. Generally, however, the Pauling electronegativity scale is used for describing the electrochemical effect in an alloy. Finally, it is shown that the Miedema parameters should be used together in order to add up to the best set. 
In pattern recognition, some item, an alloy in the present case, is positioned in a multidimensional feature space, spanned by all physical and chemical data, called features, of that particular item (the parameters given in Table 1). When several items in that feature space cluster together, it is obvious that their chemical and physical behaviour is similar. In pattern recognition, it is assumed that such behaviour not only holds for the known physical and chemical data, but also reflects similar behaviour of properties that have not been measured or cannot be measured without considerable effort.

When a large number of parameters is involved, it is important to obtain an indication about their mutual dependence. This is reflected by the correlation coefficient $c_{i j}$ :

$c_{i j}=\sum_{k=1}^{n}\left(x_{i, k}-\bar{x}_{i}\right) \cdot\left(x_{j, k}-\bar{x}_{j}\right) /\left[\sum_{k=1}^{n}\left(x_{i, k}-\bar{x}_{i}\right)^{2} \sum_{k=1}^{n}\left(x_{j, k}-\bar{x}_{j}\right)^{2}\right]^{1 / 2}$

where $x_{i, k}$ denotes parameter $i$ of item $k$, and $\bar{x}_{i}$ the mean value of parameter $i$ over the $n$ items. This can be used to reduce the dimensionality of the feature space by removal of those parameters that are highly correlated to others. Such reduction is advantageous because it simplifies the description of the property investigated for the items (here, the sign of the heat of formation of alloys).

The alloys are divided into two categories on the basis of their known chemical and physical behaviour (positive or negative heat of formation). Then the procedure WEIGHT can be applied in order to investigate which parameters are mainly responsible for the discrimination of the categories. The variance weight $(W V)$ is the ratio of the interclass variance (a measure of the spread of a certain parameter over both categories) to the intraclass variance (a measure of the spread of that parameter within one category):

$$
\begin{gathered}
W V_{j, 1,2}=\left[\sum_{k=1}^{n_{1}} \frac{x_{k, 1, j}^{2}}{n_{1}}+\sum_{k=1}^{n_{2}} \frac{x_{k, 2,1}^{2}}{n_{2}}-2 \frac{\sum_{k=1}^{n_{1}} x_{k, 1, j} \cdot \sum_{k=1}^{n_{2}} x_{k, 2, j}}{n_{1} n_{2}}\right] / \\
\times\left[\sum_{k=1}^{n_{1}} \frac{\left(x_{k, 1, j}-\bar{x}_{1, j}\right)^{2}}{n_{1}}+\sum_{k=1}^{n_{2}} \frac{\left(x_{k, 2, j}-\bar{x}_{2, j}\right)^{2}}{n_{2}}\right]
\end{gathered}
$$

where $W V_{j, 1,2}$ denotes the weight of a parameter $j$ for separating category 1 from category $2, x_{k, 1, j}$ is parameter $j$ of the $k$ th item in category 1 , and $\bar{x}_{1, j}$ is the mean value of parameter $j$ over the $n_{1}$ items in category 1 .

The procedure SELECT chooses the parameter with the largest variance weight and consecutively decorrelates all remaining parameters from the selected one by means of some kind of Gram-Schmidt procedure. These decorrelated parameters are then reweighted and so on. A final test for the 
separability of the categories is offered by LEAST, that aims to predict the category number by means of a linear combination of the chosen parameters:

$P_{k}=b_{0}+\sum_{i=1}^{n^{\prime}} b_{i} x_{i, k}$

where $P_{k}$ denotes the predicted category number of item $k, x_{i, k}$ is the $i$ th parameter of item $k$, and $n^{\prime}$ is the number of parameters; $b_{i}$ are the coefficients of the least-squares multilinear regression. Then the $P$ value is calculated which separates the two categories optimally. The percentage of correctly classified elements is called the predictive ability.

\section{SELECTION OF PARAMETERS}

Miedema et al. [5] described the heat of formation of a binary alloy, $\Delta H$, in terms of the expression

$\Delta H=f(c)\left[-P\left(\Delta \phi^{*}\right)^{2}+Q_{0}\left(\Delta n_{\mathbf{w} s}^{1 / 3}\right)^{2}\right]$

where $f(c)$ is a function of the concentration of the metals, $\phi^{*}$ and $n_{\mathrm{ws}}$ denote the electronic work function of a pure metal and the electron density at the boundary of the Wigner-Seitz cell, respectively, $\Delta$ represents the difference between the considered quantities of both models, and $P$ and $Q_{0}$ are constants. It follows directly that, in a graphical representation of $\Delta \phi^{*}$ versus $\Delta n_{\mathrm{w} s}^{1 / 3}$, the alloys with a positive heat of formation, $\Delta H>0$, are separated from the alloys with a negative heat of formation, $\Delta H<0$, by the line

$\Delta n_{\mathrm{ws}}^{1 / 3}=\left(P / Q_{0}\right)^{1 / 2} \Delta \phi^{*}$

Plots of $\Delta \phi^{*}$ versus $\Delta n_{\mathrm{ws}}^{1 / 3}$ are shown in Fig. 1 and $2 \mathrm{~A}$.

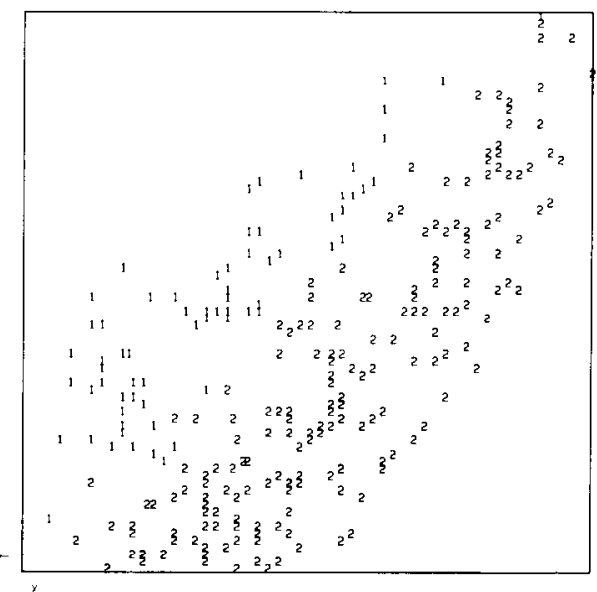

Fig. 1. $\Delta n_{\mathrm{wS}}^{1 / 3}(Y)$ versus $\Delta \phi^{*}(X)$ for 257 alloys of transition and noble metals. The alloys with positive heat of formation (category 1 ) are well separated from those with a negative heat of formation (category 2). 

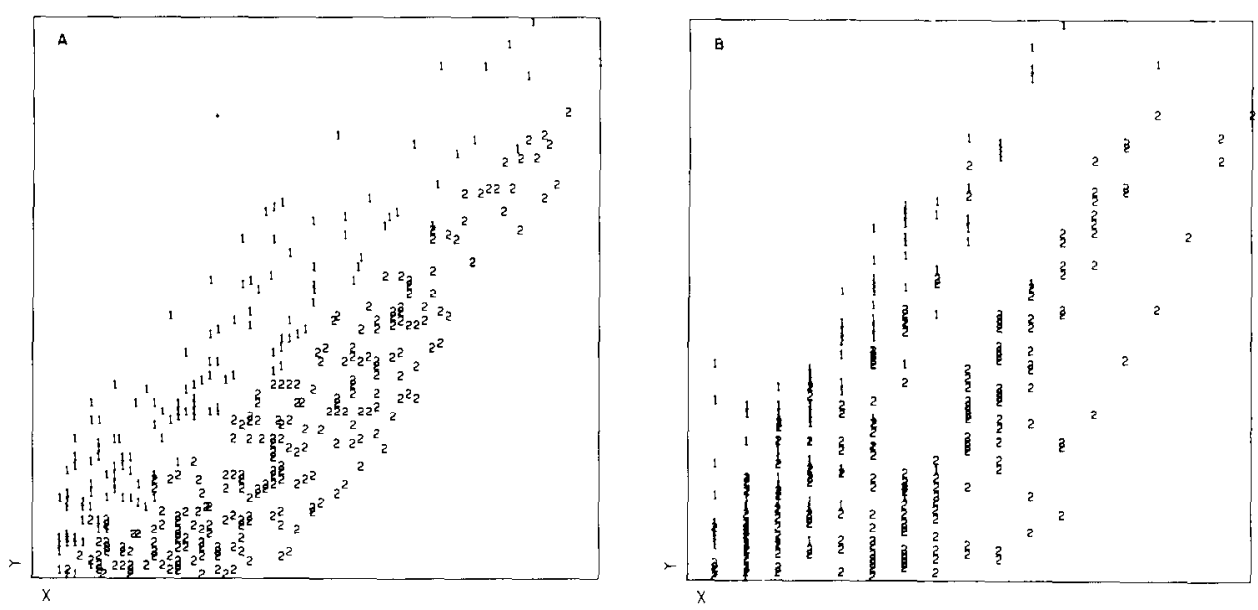

Fig. 2. A, $\Delta n_{\mathrm{ws}}^{1 / 3}(Y)$ versus $\Delta \phi^{*}(X)$ for all 390 alloys to which Eqn. (4) is applicable; the separation between category $1(\Delta H>0)$ and category $2(\Delta H<0)$ is convincing. $\mathrm{B}$, $\Delta n_{\mathrm{ws}}^{1 / 3}(Y)$ versus $\Delta x_{\mathrm{p}}(X)$, the difference in Pauling electronegativity; the categories overlap.

The $\phi^{*}$ values are based on the work function $\phi$ of the pure metals. They are obtained by slightly changing the original $\phi$ values in order to enhance the applicability of Eqn. (4). These adjustments are such that they fall within the experimental error of the $\phi$ values, which themselves represent an average of the measured orientation-dependent work functions.

The numerical value of $n_{\mathrm{ws}}$ is less easily obtained. For alkali metals, the value of $n_{\mathrm{ws}}$ can be assumed to be equal to the number of valence electrons per atomic volume. For some other elements, its value can be calculated by means of self-consistent calculations of band structure. These $n_{\mathrm{ws}}$ values appear to be highly correlated with $\left(K / V_{\mathrm{m}}\right)^{-1 / 2}$ [15], where $K$ and $V_{\mathrm{m}}$ denote the compressibility and the molar volume, respectively. Because of this high correlation, Miedema et al. [15] assumed $\left(K / V_{\mathrm{m}}\right)^{-1 / 2}$ to be the measure for $n_{\mathrm{ws}}$ but they allowed small shifts in the resulting value of $n_{\mathrm{ws}}$ in order to optimize their model. From a physical point of view, the meaning of a parameter obtained in such a way is rather obscure. Therefore a variety of parameters (Table 1) was tested for 49 pure metals (Table 2) with regard to their relevance to the alloying behaviour of two metals. The same set of parameters was recently tested for its relevance to superconducting behaviour [16]. The parameters describing the alloy are the differences between the corresponding parameters of the two constituting elements.

Information about the sign of $\Delta H$ was available for 390 alloys, where Eqn. (4) can be applied. This information was obtained from phase diagrams and from Hultgren et al. [17]; the phase-diagram information was kindly provided by Miedema and Niessen. In the first instance, only alloys consisting of metals in columns 3-11 of Table 2 are considered. The $\Delta H$ data of 257 phase diagrams out of the 378 possible combinations in this set were 


\section{TABLE 1}

Parameters investigated for their predictive ability with respect to alloying behaviour

Parameter

Symbol

1 Electronic work function as given by Miedema et al.

2 Electron density at the boundary of the Wigner-Seitz cell as given by $n_{\mathrm{ws}}^{1 / 3}$ Miedema et al.

3 Electron density at the boundary of the Wigner-Seitz cell, calculated $\quad n_{\mathrm{ws}}^{* 1 / 3}$ from compressibility and molar volume

4 Number of relevant valence electrons as given by Miedema et al. $\quad N$ rounded off to integer values

5 Specific heat at $25^{\circ} \mathrm{C}$

6 Melting point $\left({ }^{\circ} \mathrm{C}\right)$

7 Boiling point $\left({ }^{\circ} \mathrm{C}\right.$ )

8 Ionic radius in the commonest oxidation state

$\phi^{*}$

9 Bond length in the solid state of the element at $25^{\circ} \mathrm{C}$

$C_{p}$

m.p.

b.p.

$I R$

10 Heat of melting

11 Heat of sublimation at $25^{\circ} \mathrm{C}$

12 Period number in the periodic system of elements

13 Young modulus

14 Compressibility

$B L$

$\Delta H_{\mathrm{m}}$

$\Delta H_{\mathrm{s}}$

$P E R$

$Y$

$\boldsymbol{K}$

15 Debye temperature as obtained from electrical resistivity measurements $T_{\mathrm{d}}$

16 Linear term in the specific heat

17 Density at $25^{\circ} \mathrm{C}$

19 Electrical resistivity at $20^{\circ} \mathrm{C}(\mathrm{ohm} \mathrm{cm})$

RHO

available. In pattern recognition, the data are organized in categories. Two categories are distinguished here. The first category contains all phase diagrams with a positive heat of formation; this means that there are no compounds, and the mutual solubility of the constituting metals is less than $10 \%$. The second category contains the phase diagrams relating to compounds

\section{TABLE 2}

Metals which are investigated with respect to alloying

(The elements in the last 4 columns are "p-type" metals [5]. The underlined elements are assigned lower electronegativity values by Miedema et al. as is to be expected from the Pauling scale)

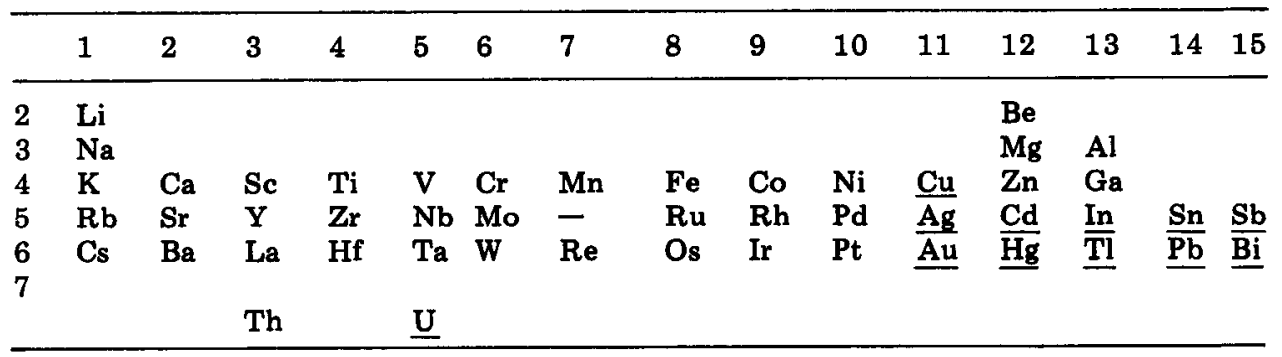


or ordered phases that are stable at low temperatures. These phase diagrams relate to alloys that are assumed to have a negative heat of formation.

The data were processed with the pattern recognition program ARTHUR [14]. Each alloy was represented as a data vector in a multidimensional space spanned by all given parameters. The aim was to look for the minimal set of parameters, which separated both categories. A suitable method of establishing the separability of two categories is offered by the LEAST program [12]. This procedure applies a multilinear least-squares regression to the category number. First, a two-parameter LEAST procedure was considered for the above-mentioned set of 257 alloys. The first parameter was always chosen to be the Miedema $\Delta \phi^{*}$ value, whereas the second parameter was taken from the set mentioned in Table 1. Moreover, a prediction was given on the basis of $\Delta \phi^{*}$ only. The results of this evaluation are presented in Table 3.

The parameters of Miedema et al. appear to be superior to all other combinations. Particularly striking is the information that is obtained when only $\Delta \phi^{*}$ is used. This demonstrates clearly the importance of the electrochemical effect in an alloy. The remaining parameters do not offer significant information concerning the alloying behaviour except for the number of valence electrons $(N)$, the bond length $(B L)$ and the compressibility $(K)$, which contribute somewhat. This is not surprising because of their high correlation with $n_{\mathrm{ws}}^{1 / 3}(0.80,-0.80$ and -0.84 , respectively). The correlation itself follows directly from the definition of $n_{\mathrm{ws}}$ as "the electron density at the boundary of the Wigner-Seitz cell" [5]. The predictive ability of the parameter set $\Delta \phi^{*}$ and $\Delta n_{\mathrm{ws}}^{1 / 3}(97.5 \%)$ differs considerably from that of the parameter set $\Delta \phi^{*}$ and $\Delta n_{\mathrm{ws}}^{* 1 / 3}(88.5 \%)$. The parameter $n_{\mathrm{ws}}^{*}$ was calculated from compressibility and molar volume data, as given by Gschneidner [18]. This parameter is the starting value for $n_{\mathrm{ws}}$. Clearly, small alterations of $n_{\mathrm{ws}}$ (see Fig. 4) strongly influence the effectiveness of the model. This point is further considered below.

\section{TABLE 3}

Predictions of a two-parameter LEAST procedure for 257 alloys of metals from columns 3-11 of Table 2. The first parameter was always $\Delta \phi^{*}$

\begin{tabular}{llll}
\hline $2^{\text {nd }}$ parameter & Predictive ability (\%) & $2^{\text {nd }}$ parameter & Predictive ability (\%) \\
\hline- & 80.5 & $\Delta\left(\Delta H_{\mathrm{m}}\right)$ & 81.0 \\
$\Delta n_{\text {ws }}^{1 / 3}$ & 97.5 & $\Delta\left(\Delta H_{\mathrm{s}}\right)$ & 78.5 \\
$\Delta$ n $_{\mathrm{W} \text { (13 }}$ & 88.5 & $\Delta P E R$ & 80.0 \\
$\Delta N$ & 83.0 & $\Delta Y$ & 81.0 \\
$\Delta C_{p}$ & 80.5 & $\Delta K$ & 85.5 \\
$\Delta$ m.p. & 80.0 & $\Delta \gamma$ & 81.5 \\
$\Delta$ b.p. & 80.0 & $\Delta \rho$ & 81.5 \\
$\Delta I R$ & 81.5 & $\Delta R H O$ & 80.5 \\
$\Delta B L$ & 83.5 & & \\
\hline
\end{tabular}


The possibility of removing $n_{\mathrm{w} s}$ and replacing it by two other parameters was then studied. This appeared to be unfruitful. The best result was obtained with the parameters $\Delta N$ and $\triangle B L$ together with $\Delta \phi^{*}$, of course (88.0\%). It should be remarked here that when the value of $N$ was increased by one unit for vanadium and chromium and decreased by one unit for uranium the predictive ability could be enhanced to $90.5 \%$. However, this was valid only for the set of 257 alloys. When these changes were extended to the entire data set of 390 alloys, the predictive ability dropped to $82.5 \%$, whereas the parameters of Miedema et al. still gave $96.0 \%$ predictive ability.

\section{COMPARISON OF THE MIEDEMA AND PAULING SCALES}

It is generally accepted that the stability of an alloy is highly correlated with the difference in electronegativity of the constituent metals. This electronegativity concept, originally introduced by Pauling [3], is well understood in principle, but hard to calculate. Electronegativity is used to estimate the ionic contribution to the heat of formation of a compound. Hodges and Stott [6] developed a theory which bears some resemblance to the model. In this theory, the electrochemical effect in an alloy is ascribed to the difference in Fermi level, $\mu$, of the constituent atomic cells. This Fermi level appears to be correlated with the Pauling electronegativity, $\chi_{p}$. The concept of electronegativity for the description of alloys is also important for the interpretation of isomer shifts in Mössbauer spectroscopy (Miedema and van der Woude [19]).

Miedema et al. preferred a physically well-defined parameter to describe charge transfer in alloys. For that purpose, an average of the orientationdependent work functions was chosen as the starting value $\phi$ for their electronegativity scale. The work function $\phi$ is strongly correlated with the Pauling electronegativity $\chi_{p}[20]$. Likewise, $\phi$ and the Fermi level are correlated [15].

The correlation coefficient for the $\phi^{*}$ scale of Miedema et al. with the Pauling $\chi_{p}$ scale is 0.94 . In Fig. 3 the relationship between $\phi^{*}$ and $\chi_{p}$ is shown. Some elements spoil the linear relationship between the two scales; without these, the correlation coefficient increases to 0.98 . In order to verify this observation, the $\Delta H$ information was divided into several groups, and the predictive ability for these groups was evaluated by the LEAST procedure using $\Delta \phi^{*}$ and $\Delta \chi_{\mathrm{p}}$ with and without $\Delta n_{\mathrm{ws}^{*}}^{1 / 3}$ The results are given in Table 4.

When the predictive abilities of $\Delta \phi^{*}$ and $\Delta \chi_{p}$ alone are compared a difference of $4 \%$ is noted for the set of 257 alloys consisting of metals from columns 3-11 of Table 2. When the 75 phase diagrams with $\mathrm{Cu}, \mathrm{Ag}, \mathrm{Au}$ and $\mathrm{U}$ are removed from this data set, both predictive abilities become equal. Clearly, the scale of Miedema et al. assigns better values to these four elements. Another striking difference concerns the predictive ability for liquid alloys consisting of " $p$-type" metals with " $p$-type", alkali, or alkaline-earth metals. 

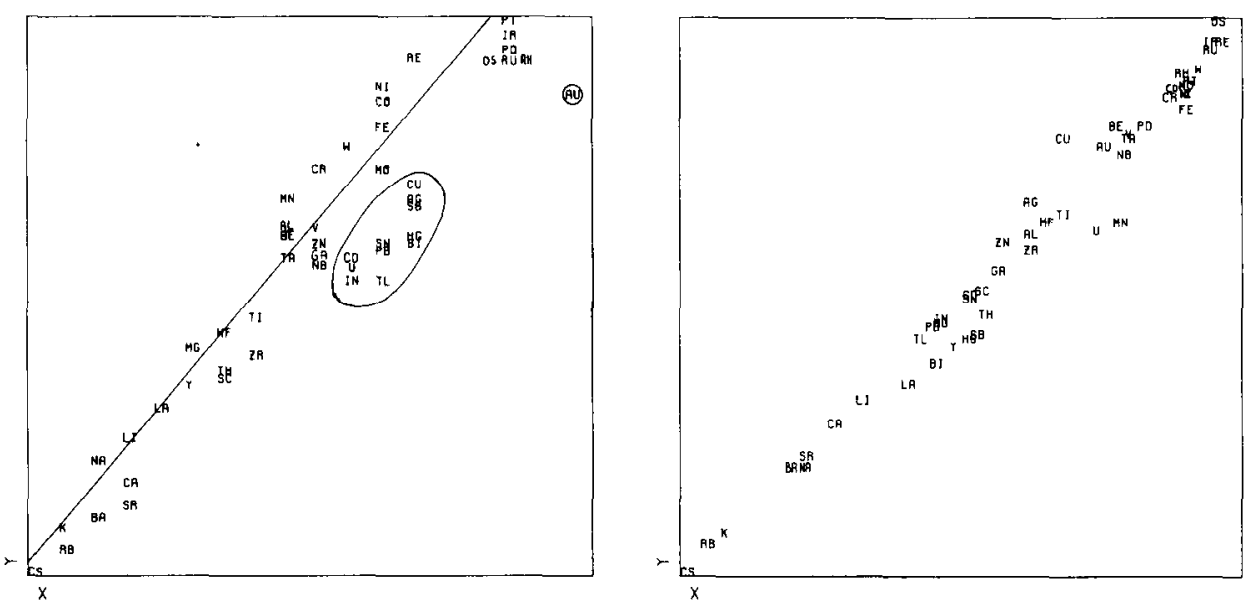

Fig. 3. The electronic work function $\phi^{*}(Y)$ for the pure elements versus electronegativity $x_{p}(X)$. The encircled elements have a significantly lower value in Miedema's scale compared to Pauling's scale. For the other elements, the least-squares line is represented by $\phi^{*}=$ $2.5 x_{p}+0.3$.

Fig. 4. $n_{\mathrm{ws}}^{* 1 / 3}(Y)$ calculated from compressibility data and molar volume versus Miedema's adjusted $n_{\mathrm{ws}}^{1 / 3}(X)$. The latter hardly differ from their starting values.

Here the charge-transfer term appears to be the only relevant parameter, because $\Delta n_{\mathbf{w s}}^{1 / 3}$ is quite small. This is clearly demonstrated by the LEAST procedure. The inclusion of $\Delta n_{\mathrm{ws}}^{1 / 3}$ does not enhance the predictive ability. The prediction based on $\Delta \phi^{*}$ is superior to that based on $\Delta \chi_{\mathfrak{p}}$.

It is concluded that Miedema et al. assigned lower values to the work functions of the elements $\mathrm{Cu}, \mathrm{Ag}, \mathrm{Au}, \mathrm{U}, \mathrm{Cd}, \mathrm{Hg}, \mathrm{In}, \mathrm{Tl}, \mathrm{Sn}, \mathrm{Pb}, \mathrm{Sb}$ and $\mathrm{Bi}$ than one would expect on the basis of the Pauling scale. These lower values allow a better description of the charge transfer in the underlying alloys. In contrast, alloys of alkali or alkaline-earth metals with metals of columns 3-10 of Table 2 are better described with the aid of the Pauling scale (i.e., on the basis of charge transfer only) so that $\phi^{*}$ might be improved. However, in combination with $\Delta n_{\mathrm{ws}}^{1 / 3}, \Delta \phi^{*}$ is distinctly superior to $\Delta \chi_{\mathrm{p}}$. This indicates that $\phi^{*}$ and $n_{\mathrm{ws}}$ are best used in combination.

The influence of replacing $\phi^{*}$ by $\phi$ in Eqn. (4) was also studied. For several $\phi$ scales, the predictive ability based on $\Delta \phi$ and $\Delta n_{\mathrm{ws}}^{1 / 3}$ was lower than $80 \%$, which is obviously inferior to the $\phi^{*}$ scale. Moreover, it was not clear which $\phi$ values should be taken.

\section{THE PARAMETERS $\phi^{*}$ AND $n_{\mathrm{ws}}$}

The decrease in predictive ability when $n_{\mathrm{ws}}$, as given by Miedema et al., was replaced by $n_{\mathrm{ws}}^{*}$ (i.e., the electron density calculated from the compressibility and the molar volume) was shown above. The plot of $n_{\mathrm{ws}}^{* 1 / 3}$ versus 
TABLE 4

LEAST predictions for one and two parameters applied to different data sets

\begin{tabular}{|c|c|c|c|c|}
\hline \multirow[t]{2}{*}{ Data set } & \multicolumn{4}{|c|}{ Predictive ability (\%) for the parameters } \\
\hline & $\Delta \phi^{*}, \Delta n_{\mathrm{wS}}^{1 / 3}$ & $\Delta \mathrm{x}_{\mathrm{p}}, \Delta n_{\mathrm{ws}}^{1 / 3}$ & $\Delta \phi^{*}$ & $\Delta x_{p}$ \\
\hline $\begin{array}{l}\text { Alloys of metals of columns } \\
\quad 3-11^{\mathrm{a}}(257)\end{array}$ & 97.5 & 85.0 & 80.5 & 76.5 \\
\hline $\begin{array}{c}\text { As above with alloys of } \mathrm{Cu}, \mathrm{Ag} \\
\mathrm{Au} \text { and } \mathrm{U} \text { removed }(\mathbf{1 8 2})\end{array}$ & 99.5 & 89.5 & 82.5 & 82.0 \\
\hline $\begin{array}{l}\text { Alloys of alkali and alkaline- } \\
\text { earth metals with a metal of } \\
\text { columns } 3-10^{2}(64)\end{array}$ & 97.0 & 92.0 & 81.5 & 89.0 \\
\hline $\begin{array}{l}\text { Liquid alloys of " } p \text {-type", } \\
\text { metals with a " } p \text {-type", } \\
\text { alkali, or alkaline-earth metal } \\
\text { (62) }\end{array}$ & 95.0 & 80.5 & 95.0 & 80.5 \\
\hline $\begin{array}{l}\text { All alloys for which Eqn. (4) } \\
\text { is valid (390) }\end{array}$ & 96.0 & 83.0 & 72.5 & 71.5 \\
\hline
\end{tabular}

${ }^{a}$ See Table 2.

$n_{\mathrm{ws}}^{1 / 3}$ given in Fig. 4 demonstrates that these parameters hardly differ, yet small changes have a significant influence as is shown in Table 5 . The influence of replacing $\Delta n_{\mathrm{ws}}^{1 / 3}$ by $\Delta n_{\mathrm{ws}}^{* 1 / 3}$ in predictions based on $\Delta \chi_{\mathrm{p}}$ instead of $\Delta \phi^{*}$ is smaller.

Table 4 shows that, although $\Delta \chi_{p}$ alone gives predictions equal to or better than $\Delta \phi^{*}$ alone for some data sets, the combination of $\Delta \phi^{*}$ with $\Delta n_{\mathrm{ws}}^{1 / 3}$ is always superior for these data sets. This is also demonstrated in Fig. 2.

It must be concluded that the work function $\phi^{*}$ and the electron density $n_{\mathrm{ws}}$, as given by Miedema et al., form an optimal set for the description of

\section{TABLE 5}

LEAST predictions indicating the influence of replacing $\Delta n_{\mathrm{ws}}^{1 / 3}$ by $\Delta n_{\mathrm{ws}}^{* 1 / 3}$

\begin{tabular}{llccc}
\hline Data set & \multicolumn{4}{l}{ Predictive ability (\%) for the parameters } \\
\cline { 2 - 5 } & $\Delta \phi^{*}, \Delta n_{\mathrm{ws}}^{1 / 3}$ & $\Delta \phi^{*}, \Delta n_{\mathrm{ws}}^{* 1 / 3}$ & $\Delta \chi_{\mathrm{p}}, \Delta n_{\mathrm{ws}}^{\mathrm{1/3}}$ & $\Delta \chi_{\mathrm{p}}, \Delta n_{\mathrm{ws}}^{* 1 / 3}$ \\
\hline $\begin{array}{l}\text { Alloys of metals of columns } \\
3-11^{\mathrm{a}}(\mathbf{2 5 7})\end{array}$ & 97.5 & 88.5 & 85.0 & 82.0 \\
$\begin{array}{l}\text { All alloys for which Eqn. (4) } \\
\text { is valid (390) }\end{array}$ & 96.0 & 87.0 & 83.0 & 78.0 \\
\hline
\end{tabular}

${ }^{2}$ See Table 2. 
alloying behaviour. It remains to be seen if this set is unique. In this context, the high correlation $(0.91)$ between $\phi^{*}$ and $n_{\mathrm{ws}}^{1 / 3}$ is surprising. As shown in Fig. 5, the $n_{\mathrm{ws}}^{1 / 3}$ values are distributed around the line $y=\left(P / Q_{0}\right)^{1 / 2} \Delta \phi^{*}$. The significant information concerning alloying behaviour is contained in the deviation from this line as noted previously $[6,7]$. The relevance of this deviation was demonstrated by application of the parameter selection procedures WEIGHT and SELECT [12].

WEIGHT selects parameters on the basis of their individual importance for the separation of two categories; this is expressed in the so-called variance weight, which is the ratio of the interclass and intraclass variance of the two categories. SELECT chooses the parameter with the highest variance weight as the most important one. The remaining parameters are then decorrelated from the chosen one and reweighted; the decorrelated parameter with the highest variance weight is selected as the second parameter and so on.

According to the WEIGHT procedure, the only important parameter of those investigated (Table 1) is $\Delta \phi^{*}$. Apart from $\Delta \phi^{*}$, SELECT assigned a significant weight to the $\Delta n_{\mathrm{ws}}^{1 / 3}$ parameter, after decorrelation from $\Delta \phi^{*}$. This means that the deviation mentioned above contains the significant information with respect to alloying behaviour.

\section{Conclusions}

Evidently the parameters $\phi^{*}$ and $n_{\mathrm{ws}}^{1 / 3}$ of Miedema et al. are the most relevant macroscopic parameters for giving a correct description of the alloying behaviour of two metals. These parameters are optimal as a set, but it remains to be seen if their values are uniquely determined for a given metal. Despite the high correlation between the two parameters, $n_{\mathrm{w} s}$ still contains additional information.

A comparison between the Pauling and Miedema scales shows that Miedema et al. assign a lower value to several elements than Pauling does. The Pauling

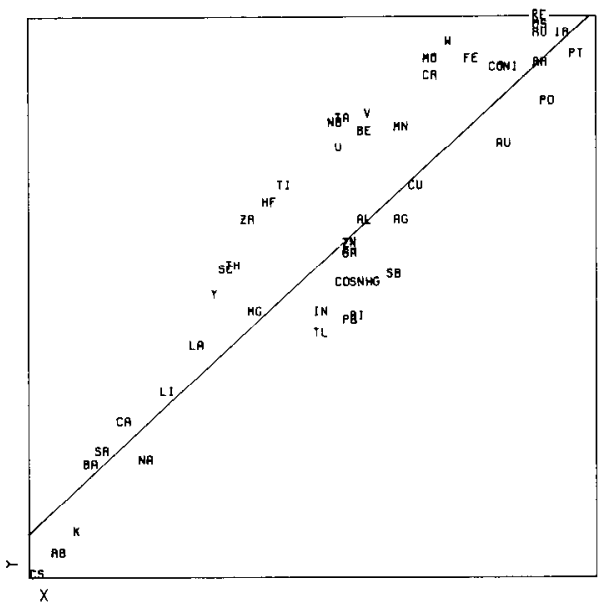

Fig. 5. The $n_{\mathrm{ws}}^{1 / 3}$ value $(Y)$ of Miedema et al. versus their electronic work function $\phi^{*}(X)$. 
scale appears to be less well suited for situations where metallic (rather than covalent) bonds compete with ionic bonds.

Pattern recognition itself does not provide theories but merely indicates the relevance of certain parameters and can be useful for developing theories. Pattern recognition is advantageous in handling large amounts of information. The influence of certain parameters can be easily studied and the correlation between parameters can be seen at first glance.

The authors thank A. R. Miedema and A. K. Niessen for stimulating and helpful discussions, for generously supplying them with the necessary data, and for critically reading the manuscript. They also thank P. F. A. van der Wiel for his valuable assistance with computer programming and B. R. Kowalski for making the ARTHUR program available.

\section{REFERENCES}

$1 \mathrm{~W}$. H. Hume-Rothery and G. V. Raynor, The Structure of Metals and Alloys, The Institute of Metals, London, 1962, p. 97.

2 J. T. Waber, K. Gschneidner, A. C. Larson and M. Y. Prince, Trans. Metal. Soc. AIME, 227 (1963) 717.

3 L. Pauling, The Nature of the Chemical Bond, Cornell University Press, Ithaca, 1960.

4 B. W. Mott, J. Mat. Sci., 3 (1968) 424.

5 A. R. Miedema, P. F. de Châtel and F. R. de Boer, Physica, 100 (1980) B1.

6 C. H. Hodges and M. J. Stott, Phil. Mag., 26 (1972) 375.

7 C. H. Hodges, J. Phys., F: Metal Phys., 7 (1977) L247; Phil. Mag., 38 (1978) 205.

8 P. F. de Châtel and G. G. Robinson, J. Phys., F: Metal Phys., 6 (1976) L173.

9 J. R. Chelikowsky and J. C. Phillips, Phys. Rev., 17 (1978) B2453.

10 A. R. Williams, C. D. Gelatt, Jr. and V. L. Moruzzi, Phys. Rev. Lett., 44 (1980) 429.

11 G. Kateman and F. W. Pijpers, Quality Control in Analytical Chemistry, Wiley, New York, 1981, Ch. 4.

12 B. R. Kowalski, Chemometrics: Theory and Application, American Chemical Society, Washington, DC, 1977, Ch. 2.

13 E. R. Malinowsky and D. G. Howery, Factor Analysis in Chemistry, Wiley, New York, 1980.

14 D. L. Duewer, J. R. Koskinen and B. R. Kowalski, ARTHUR Laboratory for Chemometrics, Department of Chemistry BG-10, Univ. of Washington, Seattle.

15 A. R. Miedema, F. R. de Boer and P. F. de Châtel, J. Phys., F : Metal Phys., 3 (1973) 1558.

16 F. W. Pijpers and G. Vertogen, J. Physique, 43 (1982) 97.

17 R. Hultgren, P. D. Desai, D. T. Hawkins, M. Gleiser and K. K. Kelly, Selected Values of the Thermodynamic Properties of Binary Alloys, American Society for Metals, Ohio, 1973.

$18 \mathrm{~K}$. A. Gschneidner, in F. Seitz and D. Turnbull (Eds.), Solid State Physics Vol. 16, Academic Press, New York, 1964, p. 275.

19 A. R. Miedema and F. van der Woude, Physica, 100 (1980) B145.

20 W. Gordy and W. J. O. Thomas, J. Chem. Phys., 24 (1956) 439. 